\title{
APPLICATION OF A STOCHASTIC REPRESENTATION IN NUMERICAL STUDIES OF THE RELAXATION FROM A METASTABLE STATE
}

\author{
FERDINANDO DE PASQUALE ${ }^{1}$, ANTONIO MECOZZI², JERZY GÓRECKI ${ }^{3}$ \\ ${ }^{I}$ INFM and Department of Physics, University of Rome "La Sapienza" \\ Piazzale Aldo Moro 2, Roma, Italy \\ ${ }^{2}$ INFMand Department of Electrical Engineering, University of L'Aquila \\ Poggio di Roio 67040, L 'Aquila, Italy \\ ${ }^{3}$ Institute of Physical Chemistry, \\ Polish Academy of Sciences and College of Science, \\ Kasprzaka 44/52, PL-01-224 Warsaw, Poland \\ ICM, Pawińskiego SA, PL-02-I06 Warsaw, Poland
}

\begin{abstract}
We study the relaxation from a metastable state using a stochastic process which is related to the generating function of the system by means of Feynman-Kac formula. The results of such representation are compared with direct numerical simulations of the stochastic differential equations describing system's evolution. We have found that the stochastic representation is more efficient from computational point of view then the direct simulations. The problems related to its numerical implementation are discussed.
\end{abstract}

\section{INTRODUCTION}

In this paper we are concerned with numerical simulations of the escape from a metastable state caused by the presence of an external noise. Such problem appears in many areas of physics and the literature on this subject is very large (see for example [1], [2]). The process of escape can be rigorously described by a Fokker-Planck equation, which gives the probability distribution of finding the system at different points of its phase space as a function of time [1]. Alternatively one may introduce a set of stochastic differential equations for the process such that the ensemble of states evolving according to these equations is characterized by the time dependent probability distribution obtained from the given by the Fokker-Planck equation. In the following we shall consider a very special case in which the stochastic differential equation describing the time evolution of our system has the form:

$$
d X=-\left(A X+B X^{2}\right) d \tau+\varepsilon d W
$$

where $X$ is the variable describing system's state and $x$ denotes time. $d W$ is the external noise (for example associated with a thermal bath) on which we assume that it is an uncorrelated Gaussian white noise. Such equation describes the evolution in the field of a cubic potential $V(x)$ characterized by two positive parameters $A$ and $B$

$$
V(x)=\frac{A X^{2}}{2}+\frac{B X^{3}}{3}
$$


and $\varepsilon$ gives the strength of the external noise $d W$. Introducing the scaled variables $x=\left(B / \varepsilon^{2}\right)^{1 / 3} X$, $t=\left(B^{2} \varepsilon^{2}\right)^{1 / 3} \tau$ and the scaled parameter

$$
a=\frac{A}{\left(B^{2} \varepsilon^{2}\right)^{1 / 3}}
$$

one may easily transform Eq. (1) to a simpler form:

$$
d x=-\left(\alpha x+x^{2}\right) d t+d W
$$

which we consider below. We study the time evolution of the system by considering the average value of $x$ as a function of time. In the simplest approach one may try to obtain it from the direct simulations of Eq. (2). Using Eq. (2) as an example we shall show that there are systems for which a stochastic representation for the generating function gives the information on system's evolution in a more efficient way than the direct simulations.

It is worth noticing that the deterministic part of Eq. (2) has interesting properties. If the noise is neglected then the system has two stationary states $\left(x_{1}=0\right.$ and $\left.x_{2}=-\mathrm{a}\right)$; the first is stable whereas the other is unstable. If initially the system is at $x(t=0) \equiv x_{0} \in(-a, \infty)$ then its evolution towards the stable stationary state is given by the formula:

$$
x(t)=\frac{a x_{0} \exp (-a t)}{a+x_{0}[1-\exp (-a t)]}
$$

and the system uniformly approaches the stable state. However if $x_{0} \in(-\infty,-a)$ than at the beginning $x(t)$ diverges to $-\infty$ and at

$$
t^{*}=-\frac{1}{a} \ln \left(\frac{a}{x_{0}}+1\right)
$$

instantaneously jumps to For $t>t^{*}$ the relaxation towards the stable state is given by the expression:

$$
x(t)=\frac{a}{\exp \left[a\left(t-t^{*}\right)\right]-1}
$$

In order to smooth out the instability at $t^{*}$ one may introduce the time average quantity $\tilde{x}$ :

$$
\tilde{x}(t)=\frac{1}{t} \int_{0}^{t} x(s) d s
$$

For the deterministic dynamics this quantity equals:

$$
\begin{array}{ll}
\tilde{x}(t)=\frac{1}{t} \ln \left\{1+\frac{x_{0}}{a}[1-\exp (-a t)]\right\}, & \text { for } t<t^{*} ; \\
\tilde{x}(t)=\frac{1}{t} \ln \left\{\frac{\left|x_{0}\right|}{a}[1-\exp (-a t)]-1\right\}, & \text { for } t>t^{*} .
\end{array}
$$

Therefore $\tilde{x}(t)$ is also divergent at $t=t^{*}$, but in a weak, logarithmic sense. 
When the thermal noise is present the system may overcome the barrier at $x=-a$, next it escapes to $-\infty$ and finally returns towards the neighborhood of the stable state from $+\infty$. Than the next escape may occur. If an ensemble of stochastic systems is concerned then, by analogy with a set of deterministic systems with continuously distributed initial conditions [3, 4], one may expect that the average over the ensemble will have a logarithmic divergence, and furthermore that the ensemble average of the time average $\langle\tilde{x}\rangle(t)$ is a continuous function of time.

The direct numerical simulations of Eq. (2) are concerned with a finite sample of systems evolving according to it. The behavior of any finite sample may be dominated by a few escaping trajectories, which contribution significantly changes the average. The lowest order algorithm for numerical simulations of Eq. (2) we use is based on the Euler-Maruyama integration scheme [5]:

$$
x(t+\Delta t)=x(t)-\left[a x(t)+x^{2}(t)\right] \Delta t+\xi \sqrt{\Delta t}
$$

where $\xi$ is a noise with zero average and unit dispersion. The accuracy of such algorithm depends on the time step used and for a typical values $(\Delta t \sim 0.0001)$ one can easily apply it when $|\mathrm{x}(\mathrm{t})|$ is small. However for $|x(t)| \gg 1$ the deterministic term becomes large and one should reduce $\Delta t$ in order to keep the desired numerical accuracy. Using such procedure the system never reaches $-\infty$ and so never "jumps" to $+\infty$ as predicted by the analytical formula for evolution.

The "jumps" may be incorporated to the numerical simulations of the system (2) if one introduces a cutoff in $x\left(x_{c}\right)$ such that the stochastic term in the dynamics may be neglected as small if compared with the deterministic one for $|x|>x_{c}$. Now one can match a numerical solution of Eq. (6) for $|x| \leq x_{c}$ with the analytical solution [Eqs. $\left.(3,4)\right]$ for $|x| \geq x_{c}$. As the result we are able to study the stochastic evolution in the neighborhood of the stable state and take into account the instantaneous jumps predicted by the deterministic dynamics.

In the numerical simulations the evolution of an ensemble of states characterized by the same initial condition is studied (we shall denote the individual trajectories by $x_{i}$ ). The average state of the system is defined as the mean value of $\left\langle x_{i}(t)>\right.$ obtained in simulations. Similarly, the ensemble average of the time average $\langle\tilde{x}\rangle$ is:

$$
\langle\tilde{x}\rangle(t)=\frac{1}{t} \sum_{i=1}^{N} \int_{0}^{t} x_{i}(s) d s
$$

where $N$ is the number of individual trajectories.

Figs. 1A, IB show $\langle x\rangle(t)$ obtained from two different stochastic simulations of an ensemble of states with the initial condition $x_{i}(t=0)=0$. The parameter $a=2$ and the results shown in $1 \mathrm{~A}$, 1B correspond to $N=100,000$ and $N=2,000,000$ trajectories respectively. The time step $\Delta t=0.0001$ and the cutoff we introduced was $x_{c}=14=7 \mathrm{a}$. One can see that after the first "escape", which happens for $t \sim 1$, the value of $\langle x\rangle$ becomes "chaotic" and the increase in the size of ensemble studied (cf. Fig. 1B) does not help in obtaining more precise information on system's 

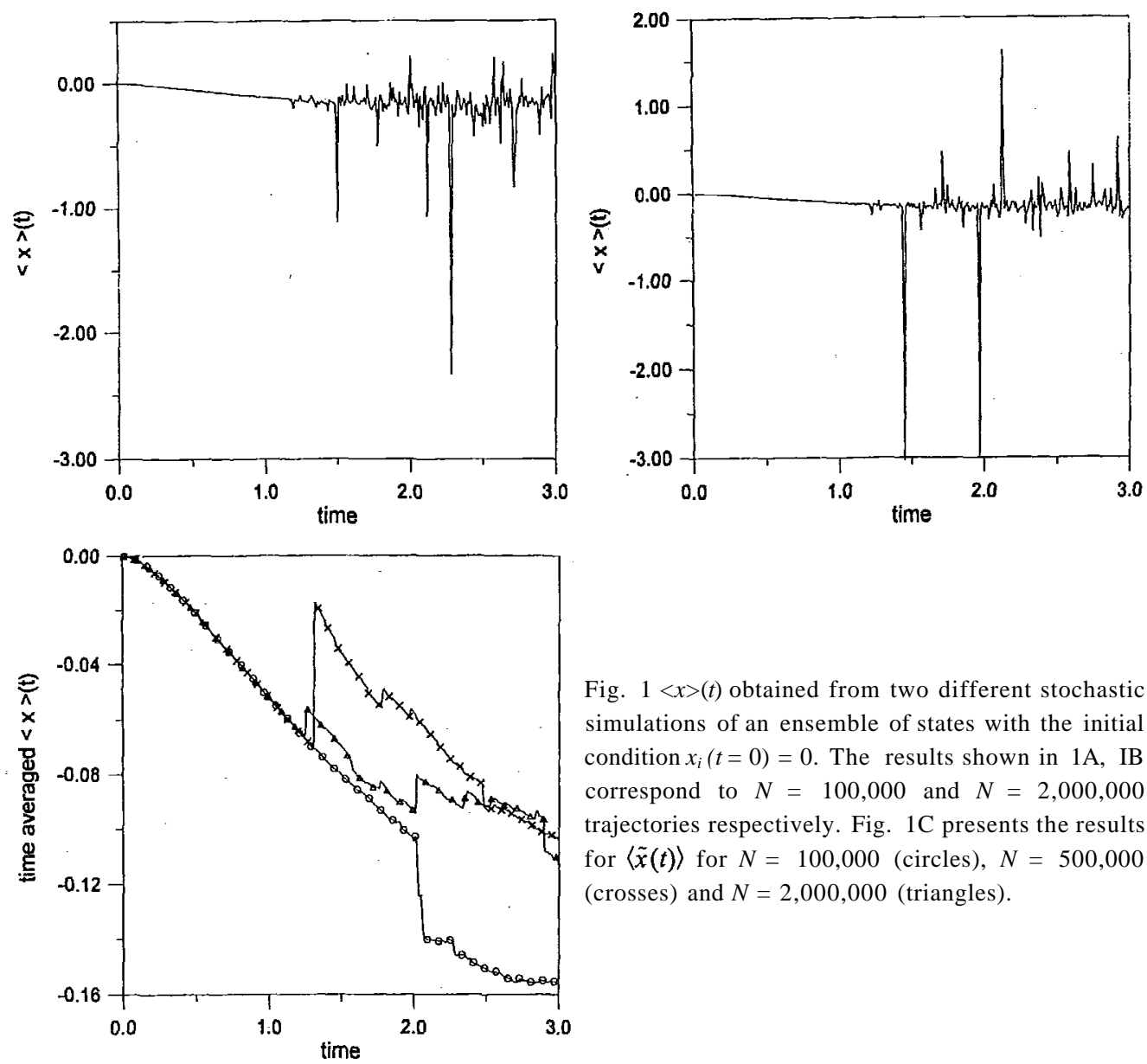

Fig. $1\langle x\rangle(t)$ obtained from two different stochastic simulations of an ensemble of states with the initial condition $x_{i}(t=0)=0$. The results shown in $1 \mathrm{~A}$, IB correspond to $N=100,000$ and $N=2,000,000$ trajectories respectively. Fig. $1 \mathrm{C}$ presents the results for $\langle\tilde{x}(t)\rangle$ for $N=100,000$ (circles), $N=500,000$ (crosses) and $N=2,000,000$ (triangles).

evolution. Here at the price of 20 times longer calculations we were able to reduce a bit fluctuations between big spikes. The time averaged quantities $\langle\tilde{x}\rangle(t)$ behave in more regular way, as shown in Fig. 1C, and the results of different simulations are quite close to one another even for the times the evolution of $\langle x\rangle$ is "chaotic". The increase in size of simulated sample from $N=100,000$ (circles) to $N=2,000,000$ (triangles) reduces the fluctuations in $\langle\tilde{x}\rangle(t)$ but it is clear that a larger statistical sample (and, of course, longer computation) is required for higher accuracy. In the next sections we show that there is another, more efficient numerical method which gives required information on the process 2 .

\section{ThE STOCHASTIC REPRESENTAtion FOR GEnERATing FunCtion}

In this section we introduce the stochastic representation for the generating function of system (2). The generating function of a stochastic process $x(t)$ can be defined as:

$$
G(\lambda, t)=\langle\exp [i \lambda x(t)]\rangle_{\xi}
$$


where the symbol \langle\rangle$_{\xi}$ means that the. average is taken over all realizations of the noise $\xi$. Knowing the generating function one can easily obtain the average value of $x$ in the stochastic process because:

$$
\langle x\rangle_{\xi}(t)=\left.\frac{\partial G(\lambda, t)}{\partial i \lambda}\right|_{\lambda=0}
$$

Assuming a discrete time increment $\Delta \mathrm{t}$ one can write down the equation for the time evolution of $G$ in the form:

$$
\begin{aligned}
G(\lambda, t+\Delta t) & =\langle\exp [i \lambda x(t+\Delta t)]\rangle_{\Delta \xi} \\
& =\left\langle\exp \{i \lambda[x(t)(1-a \Delta t)+\Delta \xi]\} \exp \left[-i \lambda x^{2}(t) \Delta t\right]\right\rangle_{\Delta \xi} \\
& =\left\langle\exp \{i \lambda[x(t)(1-a \Delta t)+\Delta \xi]\} \times\left\langle\exp \left[x(t)\left(-i \lambda \Delta u+\Delta u^{*}\right)\right]\right\rangle_{u}\right\rangle_{\Delta \xi}
\end{aligned}
$$

where $\Delta u$ and $\Delta u^{*}$ are complex conjugate noises, independent for different $t$, with the following properties: $\left(\left(\Delta u_{t}\right)^{2}\right)_{u}=\left(\left(\Delta u_{t}^{*}\right)_{u}=0,\left(\Delta u_{t} \Delta u_{t}{ }^{*}\right) u=\Delta t\right.$ and the average $<>_{u}$ denotes the average over different realizations of the process $u$. Therefore, the time evolution of the generating function can be written as

$$
G(\lambda, t)=\langle\exp [i \lambda y(t)]\rangle_{\Delta \xi, u},
$$

where the stochastic process $y(t)$ satisfies the equation

$$
y(t+\Delta t)=y(t)\left(1-a \Delta t-\Delta u_{t}+\frac{\Delta u_{t}^{*}}{i \lambda}\right)+\Delta \xi(t),
$$

with initial condition $y(0)=x_{0}$. Solution of Eq. (11) in the discretized time axis $t_{k}=k \Delta t$ [for simplicity, we use the notation $f(k)=f\left(t_{\mathrm{k}}\right)$ ], is

$$
i \lambda y(k)=v(k, 0) x_{0}+\sum_{j=1}^{k} v(k, j) \Delta \xi(j-1), \quad k \geq 1,
$$

where the auxiliary process $v$ is defined as

$$
v(k, j)=i \lambda \prod_{l=j}^{k-1}\left(1-a \Delta t-\Delta u_{k}+\frac{\Delta u_{k}^{*}}{i \lambda}\right) .
$$

It is possible to show that Eq. (13) is equivalent to the following stochastic differential equation for $v$

$$
v(k, j-1)-v(k, j)=-[a \Delta t+\Delta u(j-1)] v(k, j)+\Delta u^{*}(j-1) .
$$

The final expression for the generating function is 


$$
G(\lambda, t)=\left\langle\exp \left[x_{0} v(t, 0)+\frac{1}{2} \int_{0}^{t} v(t, s)^{2} d s\right]\right\rangle_{u}
$$

The presented approach can be obtained by a straightforward generalization of the Feynman-Kac formula [6, 7], which shows how to construct an auxiliary stochastic process, which returns the identical generating function as the original one. Equation (14) is a linear stochastic differential equation and is easily solved. The result is

$$
v(t, s)=i \lambda \exp \left[-a(t-s)-\int_{s}^{t} d u_{r}\right]+\int_{s}^{t} \exp \left[-a(t-q)-\int_{q}^{t} d u_{r}\right] d u_{q}^{*}
$$

Now we can substitute Eq. (16) into Eq. (15) and calculate the derivative over the variable $i \lambda$ at $\lambda=0$. The formula for $\langle x\rangle(t)$ becomes especially simple if at the beginning the system is at its stable stationary state $\left(x(t=0)=x_{0}=0\right)$. Then

$$
\begin{aligned}
\langle x\rangle(t) & =\left.\frac{\partial}{\partial i \lambda} G(\lambda, t)\right|_{\lambda=0} \\
& =\left\langle\exp \left[-\frac{1}{2} \int_{0}^{t} v(t, s)^{2} d s\right]\right. \\
& \times\left.\left.\int_{0}^{t} \exp \left[-a(t-s)-\int_{s}^{t} d u_{r}\right] v(t, s) d s\right|_{u}\right|_{\lambda=0}
\end{aligned}
$$

The average over processes $u$ can be calculated numerically if one considers a reasonably large sample of stochastic processes $v(t, s)$ obtained for different realizations of the noise $u$. The values of $\langle\tilde{x}\rangle(t)$ come form numerical integration of $\langle x\rangle(t)$.

\section{NUMERICAL APPLICATION OF THE STOCHASTIC REPRESENTATION FOR GENERATING FUNCTION}

In the following we shall discuss the numerical efficiency of the stochastic representation for the generating function. For numerical simulations we considered the system discussed in the Introduction [Eq. (2), with $a=2$ ], In order to apply formula (17) we need to generate an ensemble of processes $v(t, s)$ for different realizations of the noise $u$. This is done using the Euier-Maruyama integration algorithm. We have found that small fluctuations in the imaginary part of the averaged noise $\left\langle\Delta u_{t}\right\rangle_{u}$ make the result unstable. In order to keep the average values of $u$ precisely equal to zero we do the following. The noise $\Delta u_{t}$ is generated as $\Delta u_{t}=\operatorname{Re}\left(\Delta u_{t}\right)$ $+i \operatorname{Im}\left(\Delta u_{t}\right)$ from the random numbers $\operatorname{Re}\left(\Delta u_{t}\right)$ and $\operatorname{Im}\left(\Delta u_{t}\right)$. Together with the original process $v_{s}^{t}$ with the noise $\Delta u_{t}$ we consider three other processes, for which the noises are equal to: $\Delta u_{t}^{(1)}$

$=\operatorname{Re}\left(\Delta u_{t}\right)-i \operatorname{Im}\left(\Delta u_{t}\right), \Delta u_{t}^{(2)}=-\operatorname{Re}\left(\Delta u_{t}\right)+i \operatorname{Im}\left(\Delta u_{t}\right)$ and $\Delta u_{t}^{(3)}=-\operatorname{Re}\left(\Delta u_{t}\right)-i \operatorname{Im}\left(\Delta u_{t}\right)$. Such enlargement in the considered number of processes does not significantly increase the computation time, as one needs to apply a time consuming generation of random numbers $\operatorname{Re}\left(\Delta u_{t}\right)$ and $\operatorname{Im}\left(\Delta u_{t}\right)$ only once for all four processes mentioned above. 
The results shown in Fig. 2 have been obtained for only 10,000 independent processes (and therefore 40,000 total). In Fig. 2A the values of $\langle x\rangle(t)$ calculated from the representation for the generating function (the thick lines) are compared with the direct simulations of Eq. (2) for $N=500,000$ processes. The time averaged quantity coming from the stochastic representation is compared with the direct simulations in Fig. 2B (marks of those results are the same as in Fig. 1C). For short times the results of both methods are in a prefect agreement. If $t>1.5$ we see that stochastic representation marked by thick dashed line diverges. This instability is related with trajectories $v(t, s)$, which, by chance move far away from 0 (notice that zero is an attractor of their evolution). The number of trajectories for the stochastic representation of the generating function is quite small and, if by a chance a trajectory which evolves far away from zero appears, its large contribution to the generating functions is not compensated by the other processes. To avoid divergency one may neglect the distant trajectories in statistics by introducing a cutoff in their modules. The thick solid line shows the calculations for the stochastic representation in which the cutoff was at the level of 5 . The appearance of a diverging trajectory can be clearly seen in Fig. 2A for $t \sim 2.2$, however after it crossed the cutoff and its contribution was neglected in statistics, the results for $\langle x\rangle(t)$ become regular again. The values for $\langle x\rangle(t)$ obtained from the stochastic representation look as a reasonable estimation of the average obtained in the direct simulations for a very large number of trajectories.

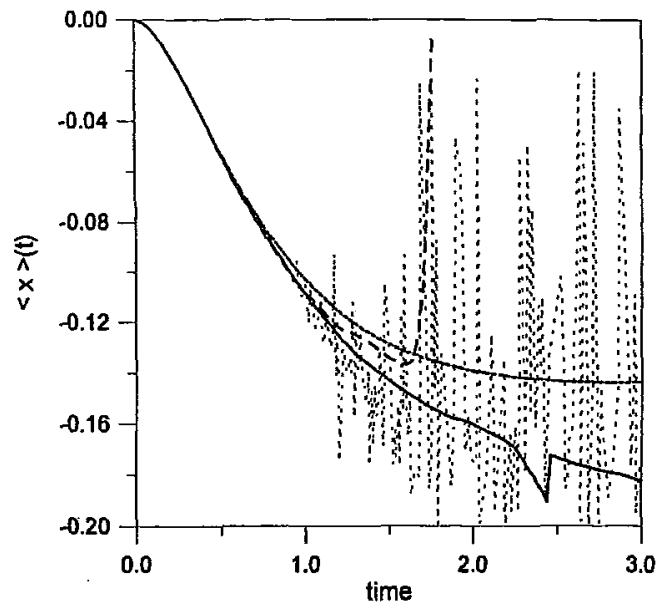

Fig. 2. The thick lines show $\langle x\rangle(t)$ (Fig. 2A) and

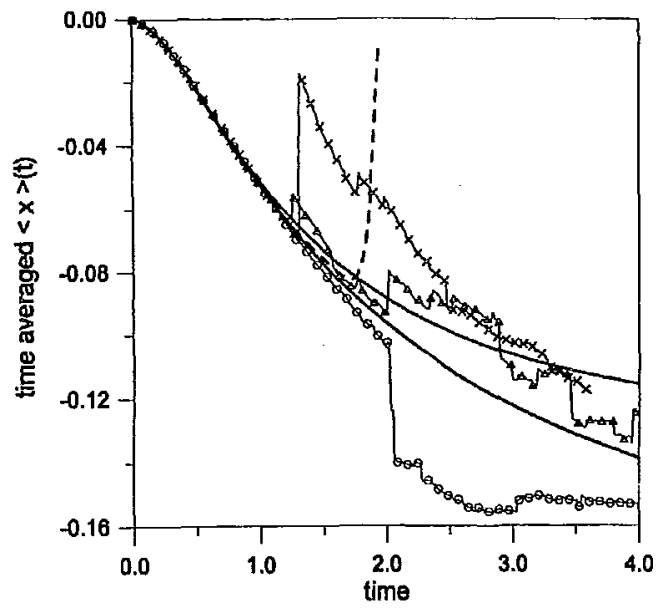

(Fig. 2B) calculated using the stochastic representation for the generating function [Eq. (17)]. The thick long dashed line, the thick solid line and the thick short dashed line correspond to no cutoff, the cutoff at the level of 5 and the cutoff at the level of 3 respectively. For comparison we present the results obtained using the direct simulations of the stochastic process (2): $\langle x\rangle(t)$ obtained for $N=500,000$ is plotted using the thin dashed line in Fig. 2A, whereas all the results of Fig. 1C are shown in Fig. 2B using the same notation

As expected, the results for $\langle\tilde{x}\rangle(t)$ are more stable and the influence of going-to-diverge trajectory can be hardly seen. Here (Fig. 2B) the stochastic representation gives much more precise information on the time dependence of $\langle\tilde{x}\rangle(t)$ than direct simulations. 
Our experience says that the value of cutoff has some influence on the results. If it is too large, it plays no role in stabilizing the process. On the other hand, if it is too small than it under estimates the absolute values of $\langle x\rangle(t)$ and $\langle\tilde{x}\rangle(t)$. The effect of small cutoff is demonstrated in Figs. $2 \mathrm{~A}$ and $2 \mathrm{~B}$, where the thick short dashed line show the results obtained for trajectories with the modules smaller than 3 .

In this paper we considered a peculiar stochastic system, for which the direct simulations do not bring precise information on its evolution, even for extremely large statistical ensemble. We demonstrated that the evolution of the same system can be precisely described by a careful application of the stochastic representation for the generating function. The stochastic representation is also more efficient from the computational point of view: 3 days of alpha CPU are required to obtain quite poor results for $N=2,000,000$, where as the stochastic representation needs just a few hours. We hope that the stochastic representation for the generating function will find many numerical applications because it is general and efficient.

\section{References}

[1] N. G. van Kampen, Stochastic processes in physics and chemistry, North-Holland, Amsterdam 1987.

[2] C. W. Gardiner, Handbook of stochastic methods, Springer, Berlin 1987.

[3] M. Frankowicz, M. Malek-Mansour, F. Baras, Physica, A146, 650-656 (1987).

[4] M. Frankowicz, Acta Phys. Polon., A74, 444-460 (1988).

[5] P. E. Kloden, Numerical solution of stochastic differential equations, Springer, Berlin 1992.

[6] C. De Witt-Morette, K. D. Elworthy, A stepping stone to stochastic analysis in: C. De Witt-Morette and K. D. Elworthy (eds.) New stochastic methods in physics, Phys. Rep., 77, 125-168 (1981).

[7] A. Mecozzi, F. de Pasquale, L. Pelti, II Nuovo Cim., 100B, $733-745$ (1987). 